\title{
LOXL2-mediated matrix remodeling in metastasis and mammary gland involution
}

\author{
Holly E. Barker ${ }^{1}$, Joan Chang ${ }^{1}$, Thomas R Cox ${ }^{1,2}$, Georgina Lang ${ }^{1,2}$, Demelza Bird ${ }^{1,2}$, \\ Monica Nicolau ${ }^{3}$, Holly R. Evans ${ }^{4}$, Alison Gartland ${ }^{4}$, and Janine T. Erler ${ }^{1,2, \#}$ \\ ${ }^{1}$ Section of Cell and Molecular Biology, The Institute of Cancer Research, London SW3 6JB, UK \\ ${ }^{2}$ Cancer Research UK Tumour Cell Signalling Unit, The Institute of Cancer Research, London \\ SW3 6JB, UK \\ 3 Department of Mathematics, Stanford University, Stanford CA 94305, USA \\ ${ }^{4}$ The Mellanby Centre For Bone Research, Department of Human Metabolism, The University of \\ Sheffield, Sheffield S10 2RX, UK.
}

\begin{abstract}
More than $90 \%$ of cancer patient mortality is attributed to metastasis. In this study we investigated a role for the lysyl oxidase related enzyme LOXL2 in breast cancer metastasis, in both patient samples and in vivo models. Analysis of a published microarray dataset revealed that LOXL2 expression is correlated with metastasis and decreased survival in patients with aggressive breast cancer. In immunocompetent or immunocompromised orthotopic and transgenic breast cancer models, we showed that genetic, chemical or antibody-mediated inhibition of LOXL2 resulted in decreased metastasis. Mechanistic investigations revealed that LOXL2 promotes invasion by regulating the expression and activity of the extracellular proteins TIMP1 and MMP9. We found that LOXL2, TIMP1 and MMP9 are co-expressed during mammary gland involution, suggesting they function together in glandular remodeling after weaning. Lastly we found that LOXL2 is highly expressed in the basal/myoepithelial mammary cell lineage, like many other genes that are up-regulated in basal-like breast cancers. Our findings highlight the importance of LOXL2 in breast cancer progression and support the development of anti-LOXL2 therapeutics for the treatment of metastatic breast cancer.
\end{abstract}

\section{Keywords}

lysyl oxidase-like 2; breast cancer; invasion; metastasis; extracellular matrix; matrix metalloproteinases; tissue inhibitors of matrix metalloproteinases; involution

\section{Introduction}

The development of therapies to treat metastatic disease has become increasingly important due to the high mortality rate associated with metastasis(1). Understanding how tumors become invasive and metastasize to secondary sites is crucial for the identification of new targets and development of new therapies for the treatment of aggressive cancers(2).

Lysyl oxidase-like 2 (LOXL2) is a member of the LOX family of extracellular matrix (ECM)-modifying enzymes comprising of LOX and LOXL1-4, all implicated in cancer

Reprint requests should be sent to Janine T. Erler, The Institute of Cancer Research, 237 Fulham Road, London SW3 6JB, UK; janine.erler@icr.ac.uk. ." corresponding author: janine.erler@icr.ac.uk. 
progression(3). LOXL2 catalyzes the cross-linking of collagens and elastin(4, 5). Increased LOXL2 expression is associated with poor prognosis in patients with colon, esophageal and squamous cell cancers $(6,7)$, and was found to promote gastric cancer metastasis(8). LOXL2 plays a role in invasion of various tumor cells(8-10) including breast cancer cells(11), and elevated LOXL2 mRNA expression was reported in highly invasive human breast cancer cell lines(12). High LOXL2 levels are associated with tumor grade in human breast cancer samples(13), found in breast carcinoma effusions(14), and negatively associated with disease-free survival in lymph-node negative breast adenocarcinomas(7). However, no previous reports have investigated the relationship between LOXL2 expression and metastasis in breast cancer patients.

In this report, we are the first to show that LOXL2 expression correlates with metastasis and poor survival in breast cancer patients. Furthermore, we demonstrate a critical role for LOXL2 in breast cancer metastasis in vivo and provide pre-clinical evidence that targeting LOXL2 is highly effective against spontaneous lung, liver and bone metastases. We show that the effects of LOXL2 on invasion are mediated through regulation of TIMP1. Finally, we provide novel data suggesting a previously uncharacterized role for LOXL2 in involution during mammary gland development, and show that LOXL2 expression is associated with basal/myoepithelial cells in the mammary gland.

\section{Materials and Methods}

\section{Patient data analysis}

We used the NKI cohort of 295 tumors(15), combined with normal tissue microarray data from Stanford Hospital(16). Of the 13 normal tissue samples, 4 were obtained from reduction mammoplasty and 9 were normal tissue from cancer patients. Expression data was first transformed using Disease Specific Genomic Analysis (DSGA)(16). We used DSGAtransformed levels of ESR1 in the cohort to identify tumors that are Estrogen Receptor negative (ER-)(ESR1 levels <-3.8 were considered to be ER-)(Supplementary Figure 1). LOXL2 levels were measured in the 72 ER- tumors, and Kaplan-Meier survival curves computed to compare tumors whose LOXL2 levels were in the top 1/3 (high) vs. the bottom $1 / 3$ (low) groups.

\section{Generation of cell lines}

4T1 mouse breast cancer cells were a kind gift from Fred Miller(17). MDA-MB-231 human breast cancer cells were obtained from ATCC. Both cell lines were authenticated using short tandem repeat (STR) analysis within the last 6 months. Cells were grown in GIBCO®DMEM media(Invitrogen) supplemented with $10 \%$ fetal calf serum. $4 \mathrm{~T} 1$ and MDA-MB-231 cells were infected with lentiviruses expressing short hairpin RNA(shRNA) libraries specific to mouse(TRC-Mm1.0) or human(TRC-Hs1.0)(Open Biosystems) LOXL2, respectively.

\section{Quantitative real-time PCR}

Total RNA was isolated from snap-frozen mammary glands or cell pellets using RNeasy Mini Kits(Qiagen). DNase treatment and cDNA synthesis were performed using QuantiTect Reverse Transcription Kits(Qiagen). Quantitative real-time PCR for mouse beta-actin, LOXL2, K14 and human beta-actin and LOXL2 was performed using TaqMan® Gene Expression Assays(Applied Biosystems).

\section{Western blotting}

Conditioned media(CM) was obtained as previously described(18). Lysates were prepared from cell pellets or mammary gland tissue in urea lysis buffer. Proteins from CM and lysates 
were separated on NuPAGE® Novex ${ }^{\circledR}$ Bis-Tris $10 \%$ gels(Invitrogen). Gels were transferred to PVDF membranes(Millipore) and probed with antibodies specific to human LOXL2, TIMP1, beta-actin(Abcam), mouse LOXL2(Santa Cruz Biotechnology Inc.) or TIMP1(R\&D Systems).

\section{LOXL2 activity assay}

Fluorescence-based enzymatic activity assays were performed as previously described(19), using 1-10 $\mu \mathrm{M}$ D-penicillamine to determine LOXL2-specific activity.

\section{In vivo assays}

For orthotopic models, control and shLOXL2 $4 \mathrm{~T} 1$ cells $\left(1 \times 10^{5}\right)$ or MDA-MB-231 cells $\left(1 \times 10^{7}\right)$ were injected into the fourth mammary fat pad of 6-8 week old female Balb/ c(Harlan) or Nude(Charles River) mice, respectively. MMTV-PyMT mice provided a transgenic model(20). Breeding pairs of these mice were kindly provided by Don White. FVB mice were used for mammary gland development studies and number of suckling pups limited to 10 for lactation and involution time-points. All experiments were approved by the Home Office and performed following UKCCCR Guidelines for the welfare and use of animals in cancer research. Treated mice received bi-weekly intraperitoneal injections of Dpenicillamine(Sigma) at $150 \mathrm{mg} / \mathrm{kg}$, anti-LOXL2 antibody(Santa Cruz Biotechnology Inc.) or IgG from goat serum(Sigma) at $0.5 \mathrm{mg} / \mathrm{kg}$ for 4 weeks. Caliper measurements of the primary tumor size were taken three times a week until a maximum size was reached at which point mice were culled. The tumors, organs and legs were removed and either fixed in $4 \%$ paraformaldehyde or flash-frozen.

Lung and liver metastases were classified as any cluster of four or more abnormal cells(21) and were quantified in sections stained with haematoxylin and eosin $(n=3$ sections per mouse). Tibias and femora were scanned using a CT scanner (model 1172; Skyscan). Images were captured every $0.7^{\circ}$ through $180^{\circ}$ rotation of the bone and reconstructed using the Skyscan Recon software to create 3D models of each bone using the Skyscan CT analysis software. Osteolytic lesions were assessed in the 3D models of the tibias and femora using an automated method and Image J software(NIH).

\section{Invasion and 3D assays}

Collagen invasion, transwell invasion and 3D growth assays were carried out as previously described(21-23). Cells were transfected with SMARTpool siRNAs specific for human LOXL2(Dharmacon). Recombinant human TIMP1(R\&D systems) was added to wells at a final concentration of $10 \mathrm{nM}$.

\section{Immunohistochemistry/Immunofluorescence}

Staining was performed as previously described $(21,24)$ using antibodies described above plus cytokeratin 14(Abcam). Alexa Fluor ${ }^{\circledR}$ secondary antibodies were used for immunofluorescence(Molecular Probes, Invitrogen).

\section{Mammary epithelial cell preparation and sorting}

Mammary epithelial cell preparation and sorting was performed as previously described(25) using anti-CD24-FITC, anti-CD45-PE-Cy5, anti-Sca-1-PE BD Biosciences antibodies.

\section{Statistical Analysis}

Data was analyzed using the Student t-test unless otherwise stated, and considered significant when the $p$ value was $<0.05$. All statistical tests were two-sided. Bar graphs 
represent the mean and standard error across independent experimental repeats unless otherwise stated. Statistical significance representations: ${ }^{*} p<0.05,{ }^{* *} p<0.01, * * * p<0.001$.

\section{Results}

\section{LOXL2 correlates with metastasis and survival}

We first performed a retrospective study to assess if LOXL2 mRNA expression levels correlate with metastasis and survival of breast cancer patients, employing a previously published dataset(15). We found that patients with estrogen receptor negative (ER-) tumors expressing high levels of LOXL2 have a significantly poorer prognosis. Moreover, LOXL2 expression significantly correlated with decreased overall survival and metastasis-free survival $(p=0.023$ and $p=0.0367$, respectively. Figures $1 \mathrm{~A}+\mathrm{B}$ ).

\section{LOXL2 is not required for primary tumor growth}

In order to investigate the role of LOXL2 in breast cancer metastasis, we chose two ERbreast cancer cell lines for our studies: human MDA-MB-231 and mouse 4T1 cells. Both cell lines expressed high levels of secreted LOXL2 protein, two forms detected in mouse $(150 \mathrm{kDa}+60 \mathrm{kDa})$ and one in human $(120 \mathrm{kDa})$ (Supplementary Figure $2 \mathrm{~B})$. Three lines of 4T1 mouse breast cancer cells stably expressing either an shRNA specific to mouse LOXL2 (4T1shLOXL2\#1 and 4T1shLOXL2\#2), or a scrambled control sequence (4T1control) were generated. In addition, two lines of MDA-MB-231 human breast cancer cells stably expressing a control shRNA (MDA-MB-231 control) or an shRNA specific to human LOXL2 (MDA-MB-231shLOXL2) were generated. All the shLOXL2 cell lines had significantly decreased expression of LOXL2 mRNA and protein compared with controls (Supplementary Figures 2A+B). Knockdown of LOXL2 in MDA-MB-231 was also achieved using siRNA (Supplementary Figure 2B). Importantly, LOXL2 knockdown reduced LOXL2 enzymatic activity (Supplementary Figure 2C). D-penicillamine, a chemical inhibitor of LOXL2, was also used to inhibit LOXL2 activity (Supplementary Figure 2C)(26).

The expression levels of LOXL1, LOXL3 and LOXL4 were unchanged in shLOXL2 cell lines (data not shown). In contrast, LOX expression appeared to be increased in 4T1shLOXL2 cells (Supplementary Figure 2D), but not in the MDA-MB-231shLOXL2 cells (data not shown). It was noted that knockdown of LOXL2 had no effect on the proliferation of 4T1 or MDA-MB-231 cells in 2D cultures (Supplementary Figure 3A).

To assess the role of LOXL2 in primary tumor formation, control and shLOXL2 cells were implanted into the mammary fat pad of mice (orthotopic site). 4T1s were injected into immunocompetent syngeneic Balb/c mice, and MDA-MB-231s were injected into immunocompromised nude mice. Half of the mice injected with 4T1 control cells received bi-weekly doses of D-penicillamine, a chemical inhibitor of LOXL2 activity(26). Mice injected with 4T1shLOXL2 cells developed mammary tumors at a faster rate than mice injected with control cells, however, no difference was observed in primary tumor growth between MDA-MB-231 control and shLOXL2 tumors (Supplementary Figure 3B). Consistent with this, treatment with D-penicillamine had no effect on 4T1 tumor growth rate (Supplementary Figure 3B). Thus, LOXL2 is not required for primary tumor growth.

\section{LOXL2 enables metastases in vivo}

Full necropsies of the mice were performed at time of sacrifice, when tumors reached maximum permitted size. Strikingly, mice bearing 4T1shLOXL2 tumors or control tumors treated with D-penicillamine developed significantly fewer lung metastases than untreated mice bearing control tumors $(p=0.044, p=0.013$ and $p=0.017$ for D-penicillamine-treated, 
shLOXL2\#1 and shLOXL2\#2, respectively; Figure 2A). Mice bearing MDAMB-231shLOXL2 tumors also developed significantly fewer lung metastases than mice bearing control tumors ( $p=0.002$; Supplementary Figure 3C).

As 4T1 tumors readily metastasize to liver and bone, we also quantified metastases to these organs. Consistent with the lung data, mice bearing shLOXL2 tumors or control tumors treated with D-penicillamine developed significantly fewer liver metastases than untreated mice bearing control tumors $(p=0.032, p=0.009$ and $p=0.010$ for D-penicillamine-treated, shLOXL2\#1 and shLOXL2\#2 tumors, respectively; Figure 2B). In addition, smaller lung and liver metastases were observed in response to LOXL2 inhibition (Figure $2 \mathrm{~A}+\mathrm{B}$ ). These mice also developed significantly fewer bone lesions ( $p=0.023$ for shLOXL2\#2 tibias, and $p=0.015$ and $p=0.007$ for shLOXL2\#1 and shLOXL2\#2 femora, respectively; Figure 2C). Mice bearing shLOXL2\#2 tumors also displayed significantly smaller lesions ( $p=0.047$ and $p=0.036$ for tibias and femora, respectively; Supplementary Figure 3D). These results suggest that LOXL2 is needed for metastatic colonization and metastatic growth.

Proliferation was found to be unchanged in tumors of mice bearing control and shLOXL2 tumors (Supplementary Figure 4A). In contrast, apoptosis was increased in shLOXL2 tumors compared to control tumors (refer: Supplementary Figure 4B). However, these changes did not affect primary tumor growth (Supplementary Figure 3B). Furthermore, no changes in proliferation or apoptosis were observed in metastatic lung tumors (data not shown). These findings suggest that LOXL2 enhances metastatic colonization and growth but does not affect proliferation or apoptosis in lesions formed.

Tumors were stained with antibodies specific for all LOX family members. Consistent with our in vitro findings, LOXL2 expression was reduced in shLOXL2 tumors (Supplementary Figure 4C), the expression levels of LOXL1, LOXL3 or LOXL4 were unchanged (data not shown), and the expression of LOX was increased in 4T1shLOXL2 tumors (Supplementary Figure 4C) but not in MDA-MB-231shLOXL2 tumors (data not shown).

We additionally tested a specific LOXL2-targeting antibody in the 4T1 syngeneic model of breast cancer metastasis. Mice were given bi-weekly injections of either a LOXL2 antibody or an isotype-matched control IgG antibody. No difference was observed in primary tumor growth rate between mice treated with the LOXL2 antibody and controls (data not shown). However, a significant decrease was observed in the number of metastases present in lungs of mice treated with LOXL2 antibody compared to controls ( $p=0.005$; Figure 2D). These results demonstrate that inhibition of LOXL2 by genetic, chemical and antibody means leads to a significant decrease in the formation of distant metastases in both immunocompetent and immunocompromised orthotopic models of breast cancer without reducing primary tumor growth, suggesting that LOXL2 plays an important role in breast cancer metastasis.

\section{Inhibition of LOXL2 in a transgenic model reduces lung metastases}

Orthotopic models involving relatively homogeneous cell lines have their limitations. Therefore, we chose to further assess the role of LOXL2 in breast cancer metastasis using the well-established MMTV-PyMT (Mouse mammary tumor virus-polyoma middle T antigen) transgenic model of ER- breast tumorigenesis(20, 27). Female MMTV-PyMT mice develop dysplastic lesions in the mammary epithelium around 3 weeks of age which progress to palpable mammary adenocarcinomas by 5-6 weeks of age and lung metastases with high penetrance by 8-9 weeks of age(20). Mice were given bi-weekly injections of Dpenicillamine from 4 weeks of age (i.e. prior to initial tumor formation) for 4 weeks, or were left untreated. No significant difference in the rate of primary tumor growth was observed (data not shown). Mice were sacrificed around 10 weeks of age and lungs analyzed for metastases. Mice treated with D-penicillamine displayed significantly fewer lung metastases 
than control mice ( $p=0.007$; Figure 2D, right panel). No differences in proliferation or apoptosis were observed in tumors or lungs of mice (Supplementary Figure 4D). Importantly, initiating treatment in mice later than 5 weeks of age had no effect on metastatic burden (data not shown), suggesting that LOXL2 is required for the early stages of metastasis.

\section{LOXL2 increases in vitro invasion}

As invasion is the first step in metastasis we tested the role of LOXL2 in invasion of 4T1 and MDA-MB-231 cells. Genetic knockdown of LOXL2 resulted in a significant decrease in invasion through collagen ( $p=0.009$ and $p=0.002$ for $4 \mathrm{~T} 1$ and MDA-MB-231 cells, respectively; Figure 3A). Similar results were obtained when cells were subjected to transwell invasion assays through Matrigel ( $p=0.001$ and $p=0.014$ for 4T1 and MDAMB-231 cells, respectively; Figure 3B). These findings suggest that LOXL2 is required for the invasive capabilities of these breast cancer cell lines.

\section{Knockdown of LOXL2 decreases TIMP1}

LOX enzymes modify the ECM, which can affect the expression and activity of other matrix remodeling enzymes, enhancing tumor cell invasion $(18,28)$. A recent study revealed a positive association between the expression of LOXL2, tissue inhibitor of metalloproteinase-1 (TIMP1) and matrix metalloproteinase-9 (MMP9) in human colorectal cancer, all were up-regulated in CRC samples relative to normal colon tissue(29). We noted reduced secreted TIMP1 protein levels in 4T1 and MDA-MB-231 shLOXL2 cells (Figure 4A), suggesting that LOXL2 regulates TIMP1 protein expression in breast cancer cells. Furthermore, immunofluorescent staining of 4T1/MDA-MB-231 control and shLOXL2 tumors for TIMP1 demonstrated that knockdown of LOXL2 resulted in decreased expression of TIMP1 (Figure 4B), confirming our in vitro findings.

\section{Recombinant TIMP1 restores invasive ability of LOXL2 knockdown cells}

We tested if TIMP1 could compensate for knockdown of LOXL2 in invasion. Recombinant TIMP1 protein was added to both control and LOXL2 knockdown cells, and cell invasion quantified. Addition of exogenous TIMP1 had no effect on the invasion of control cells. The LOXL2 knockdown cells exhibited a significantly decreased level of invasion as previously observed ( $p=0.023$; Figure $4 \mathrm{C}$ ). Importantly, TIMP1 was able to significantly restore the invasive capability of LOXL2 knockdown cells to the same level as control cells ( $p=0.032$; Figure 4C), confirming a role in mediating invasion downstream of LOXL2.

MDA-MB-231 cells form invasive branching structures when grown in 3D matrix(21). We noted that LOXL2 knockdown cells formed round structures with fewer protrusions compared to control cells. Addition of exogenous TIMP1 protein to LOXL2 knockdown cells restored the ability of these cells to form protrusions in 3D Matrigel cultures (Figure 4D). Similar results were observed when cells were grown in 3D collagen cultures (Supplementary Figure 5A). These results confirm TIMP1 as a downstream mediator of LOXL2-driven invasion.

\section{Knockdown of LOXL2 decreases MMP9}

Although TIMP1 targets and inhibits the matrix metalloproteinase MMP9, it has been proposed that MMPs and TIMPs are co-regulated and concurrently have been found to be up-regulated in many tumor samples relative to normal tissue(30,31). To assess the activity of MMP9 in control and shLOXL2 4T1/MDA-MB-231 cells gelatin zymography was performed. Knockdown of LOXL2 in 4T1/MDA-MB-231 cells resulted in decreased MMP9 activity (Supplementary Figure 5B), suggesting that LOXL2 also regulates the activity of 
MMP9. Furthermore, immunofluorescent staining of tumors for MMP9 demonstrated that knockdown of LOXL2 resulted in decreased expression of MMP9 (Supplementary Figure $5 \mathrm{C})$, confirming our in vitro findings.

\section{High LOXL2 expression and activity during mammary gland involution}

Deregulation of normal signaling processes during morphogenesis can lead to tumorigenesis. Many cellular processes that control normal mammary gland development, such as tissue invasion, are characteristics of cancer progression. To gain further insight into the mechanism of action of LOXL2 in breast cancer progression, we analyzed LOXL2 expression during normal mammary gland development.

Samples were prepared from mammary glands harvested from FVB mice at different stages of mammary gland development. LOXL2 mRNA was expressed throughout development with highest expression during the late pregnancy (Figure 5A). The 150kDa LOXL2 protein was expressed at all stages of mammary gland development (Supplementary Figure 6A), however, highest levels of the $60 \mathrm{kDa}$ LOXL2 protein were exhibited during lactation and involution (Figure 5B). As LOXL2 is a matrix-modifying enzyme, these results suggest that LOXL2 plays a role in the remodeling of the mammary gland that occurs during involution.

Expression of the other LOX family members during mammary gland development was also analyzed. No change in expression of LOXL1, LOXL3 or LOXL4 was observed (data not shown). In contrast, LOX appeared to be most highly expressed during pregnancy and lactation (Supplementary Figure 6A).

\section{TIMP1 is associated with LOXL2 during involution}

TIMPs and MMPs have been found to play roles in matrix remodeling during mammary gland development $(32,33)$. Therefore, we investigated the expression of TIMP1 during the various stages of mammary gland development. Expression of TIMP1 could only be detected during involution (Figure 5B), when LOXL2 activity was highest (Figure 5C). Active MMP9 was mostly associated with LOXL2 however not as tightly as TIMP1 (Supplementary Figure 6A).

We analyzed the expression of MMP2 and MMP14, two additional matrix-modifying enzymes found to be up-regulated in invasive breast cancer(34). While their expression was varied throughout mammary gland development (Supplementary Figure 6A), they were not associated with LOXL2 activity, consistent with analysis of their expression in our control and shLOXL2 cells lines (data not shown). Therefore, TIMP1 appears to be specifically associated with LOXL2 expression and activity. It is likely that LOXL2 plays a role in modifying the mammary gland during involution not only through its action as a collagen cross-linker, but also through regulation of TIMP1.

\section{LOXL2 is expressed in basal epithelial and stromal cells}

To assess the mammary cell types expressing LOXL2, sections from different stages of mammary gland development were immunostained for LOXL2. Consistent with western blotting results, LOXL2 protein was expressed at all stages of development (Supplementary Figure 6B). LOXL2 staining appeared cytoplasmic in the basal epithelial cells surrounding the luminal ductal epithelial cells (Figure 6A). LOXL2 expression was also high in the stromal cells of the mammary gland (Supplementary Figure 6B).

\section{LOXL2 is expressed in the basal/myoepithelial cell lineage}

Understanding the relationship between normal epithelial cell types and different disease states is required to provide insight into the specific cell types predisposed to carcinogenesis. 
Therefore, we analyzed the expression of LOXL2 in the three main cell lineages in normal mammary epithelium: the basal/myoepithelial, luminal ER+ and luminal ER- epithelial cell populations $(25,35)$. Primary mouse mammary epithelial cells were sorted into CD24+/Low/ Sca-1 ${ }^{-}$(basal/myoepithelial) CD24 ${ }^{+/ \mathrm{High}} / \mathrm{Sca}^{-1^{-}}$(ER-) and CD24+/High $/ \mathrm{Sca}-1^{+}(\mathrm{ER}+)$ populations (Supplementary Figure 6C), and relative LOXL2 expression levels determined. Consistent with LOXL2 immunostaining, LOXL2 was highly expressed in the basal/ myoepithelial cell population, compared with the luminal epithelial populations which exhibited very low levels of $L O X L 2$ (Figure 6C). The isolation of the basal/myoepithelial population was confirmed by analysis of the expression of two basal cell markers, Keratin14 (K14) and p63 (Figure 6B+Supplementary Figure 6D).

To further confirm expression of LOXL2 in basal epithelial cells, mouse mammary gland sections were co-stained for LOXL2 and K14. Many basal cells (K14+) were found to also express LOXL2 (Figure 6C). Finally, we analyzed our primary tumors and found that 4T1 shLOXL2 tumors exhibited reduced expression of the basal markers K14 and p63 (Figure 6D+Supplementary Figure 6D), confirming that high LOXL2 expression is associated with maintaining a more aggressive phenotype.

\section{Discussion}

Over 40,000 women die from metastatic breast cancer in the US each year(36). Once metastases are detected, median survival is only 20 to 24 months(37). Thus, it is imperative that novel therapeutic strategies are developed and adopted for the treatment of patients with highly aggressive breast cancer.

Here, we show that high LOXL2 expression is correlated with decreased overall and metastasis-free survival in a subset of patients with aggressive breast cancer (ER-tumors). Therefore, LOXL2 expression may have prognostic value in determining which patients are most likely to develop metastatic disease. Furthermore, we analyzed the in vivo role of LOXL2 using orthotopic and transgenic mouse models of breast cancer, and demonstrated that the size and number of distant metastases formed was significantly reduced. These results suggest that LOXL2 inhibitors should be considered in the development of new therapies for the treatment of metastatic breast cancer.

Despite its critical role in enabling metastasis, LOXL2 was found not to be essential for cell proliferation and primary tumor growth. In fact, growth was not significantly different for all treatments, save one exception: 4T1shLOXL2 tumors were found to consistently have a faster growth rate than control tumors, which could not be attributed to changes in proliferation or apoptosis. As this effect was not observed when mice bearing 4T1 tumors were treated with a chemical and antibody inhibitor, nor in mice bearing MDAMB-231shLOXL2 tumors compared with controls, we propose this could be due to increased LOX expression we observed in these cells only, as LOX has previously been shown to play a role in primary tumor growth in a breast cancer model(38).

We have previously shown that LOX is essential for breast cancer metastasis through effects on tumor cell invasion and metastatic colonization $(24,39)$. Inhibition of LOX prevents $\mathrm{CD} 11 \mathrm{~b}+$ cell recruitment required for pre-metastatic niche formation and reduces lung foci formation in tail vein metastasis assays $(21,24)$. However, unlike LOX, LOXL2 inhibition did not affect CD11b cell recruitment to the lungs or the number of lung foci formed (data not shown), suggesting that LOXL2 is not required for the initial formation of metastases. This is supported by the MMTV-PyMT model where we noted that later inhibition of LOXL2, at a stage where tumor cells had already disseminated, did not reduce the number of metastases. However, the size of metastases and lung foci were reduced with LOXL2 
inhibition in our models, despite unaltered proliferation and apoptosis, suggesting some LOXL2-dependency for metastatic growth although the mechanisms are subject to further investigation.

In the present study we found that LOX expression levels were not changed in response to LOXL2 inhibition (except increased in 4T1shLOXL2 cells, see above), however, invasion and metastasis were still suppressed in vitro and in vivo, suggesting that these enzymes cannot compensate for each other. Altered expression of the other LOX family members has been observed in various tumor types compared to normal tissue(3), however, we found no change in the expression of LOXL1, LOXL3 or LOXL4 in response to LOXL2 inhibition, suggesting they are not involved in LOXL2-mediated invasion and metastasis in our models.

A relevant paper on LOXL2 was published while our manuscript was under revision. BarryHamilton et al(40) showed that inhibition of LOXL2 using a monoclonal antibody significantly reduced bone metastases from intracardiac injection of MDA-MB-231, as we observed in our spontaneous 4T1 model. In contrast, the authors observed a significant reduction in primary tumor growth, which we did not observe in all three models we tested in response to genetic, chemical or antibody inhibition of LOXL2. However, this difference may be explained by different specificities of their LOXL2-targeting antibody.

We carried out in vitro assays to determine how LOXL2 drives the early stages of metastasis. We found that knockdown of LOXL2 decreased invasion and branching morphogenesis in 3D cultures. These findings support a role for LOXL2 in the early stages of metastasis, and support previous reports suggesting a role for LOXL2 in invasion(8-11). To understand the mechanism by which LOXL2 increases invasion of tumor cells we studied other enzymes involved in remodeling of the ECM. We observed decreased TIMP1 and MMP9 expression in vitro and in vivo in response to LOXL2 knockdown. TIMP1 was found to functionally compensate for LOXL2 knockdown in invasion and branching assays. Cross-linking of collagens is known to activate enzymes involved in matrix remodeling, such as MMPs $(18,24)$. We propose that this is the mechanism by which LOXL2 increases TIMP1 and MMP9, thereby increasing degradation/remodeling of the ECM and enabling subsequent metastatic dissemination.

We examined normal mammary gland development to gain further insight into the mechanism of action of LOXL2. Breakdown of the basement membrane and ECM remodeling are prominent features of mammary gland involution(41). Furthermore, processes that are known to promote tumorigenesis, play major roles in modifying the mammary microenvironment during involution $(42,43)$. LOXL2 was expressed during all stages of mammary gland development but was most active in the involuting mammary gland when extensive ECM remodeling is taking place. Importantly, we also observed high TIMP1 and MMP9 during involution, providing further evidence that LOXL2 activity regulates the expression of these proteins involved in matrix remodeling.

Recently, a mouse mammary gland involution mRNA signature was found to predict breast cancers with high metastatic activity in the NKI 295 microarray dataset we used for our initial prognostic study(44). Not only did Stein et al(44) link genes associated with involution to breast cancer metastasis, but also genes associated with hypoxia and copper ion homeostasis, two important regulators of $\operatorname{LOXL2}(45,46)$. Furthermore, we also show that LOXL2 is expressed in mammary stroma and basal epithelial cells surrounding the mammary ducts. Our analysis of the three main cell lineages in the mammary gland confirmed that LOXL2 is expressed in the basal/myoepithelial compartment. Cells in this population express many genes common to basal-like tumors, the most aggressive form of breast cancer(47, 48). Finally, we found reducing the expression of LOXL2 in basal tumors 
led to a decrease in the expression of the basal markers K14 and p63. Taken together, these data support our observations that LOXL2 expression is elevated in the involuting mammary gland, in aggressive breast cancer cell lines, in patients with poor prognosis, and facilitates the progression of breast cancer.

In conclusion, our data show that LOXL2 is a potent mediator of breast cancer metastasis through effects on tumor cell invasion and regulation of matrix remodeling proteins. These same proteins are associated with LOXL2 activity in the involuting mammary gland. We show that LOXL2 correlates with poor prognosis in breast cancer patients, and provide preclinical evidence that targeting LOXL2 is effective against breast cancer metastases. These findings clearly demonstrate that LOXL2 is an excellent therapeutic target against metastatic breast cancer.

\section{Supplementary Material}

Refer to Web version on PubMed Central for supplementary material.

\section{Acknowledgments}

The authors thank Andreas Hadjiprocopis for assistance with statistics, Kay Savage for histopathology, Fredrik Walberg for assistance with flow cytometry, Samra Turajlic and Grazia Saturno for STR analysis, and Clare Isacke and Matt Smalley plus lab members at ICR for assistance. We also thank the following for financial support: The Institute of Cancer Research(HEB, JTE), the Breast Cancer Campaign(ref 2008NovPhD17, JC), Cancer Research UK(ref C107/A10433, TRC, GL, DB, JTE). All in vivo experiments were approved by the Home Office and performed following UKCCCR Guidelines for the welfare and use of animals in cancer research.

\section{References}

1. Steeg PS. Tumor metastasis: mechanistic insights and clinical challenges. Nat Med. 2006; 12:895904. [PubMed: 16892035]

2. Gupta GP, Massague J. Cancer metastasis: building a framework. Cell. 2006; 127:679-95. [PubMed: 17110329]

3. Payne SL, Hendrix MJ, Kirschmann DA. Paradoxical roles for lysyl oxidases in cancer--a prospect. J Cell Biochem. 2007; 101:1338-54. [PubMed: 17471532]

4. Csiszar K. Lysyl oxidases: a novel multifunctional amine oxidase family. Prog Nucleic Acid Res Mol Biol. 2001; 70:1-32. [PubMed: 11642359]

5. Kim YM, Kim EC, Kim Y. The human lysyl oxidase-like 2 protein functions as an amine oxidase toward collagen and elastin. Mol Biol Rep. 2010

6. Fong SF, Dietzsch E, Fong KS, et al. Lysyl oxidase-like 2 expression is increased in colon and esophageal tumors and associated with less differentiated colon tumors. Genes Chromosomes Cancer. 2007; 46:644-55. [PubMed: 17394133]

7. Peinado H, Moreno-Bueno G, Hardisson D, et al. Lysyl oxidase-like 2 as a new poor prognosis marker of squamous cell carcinomas. Cancer Res. 2008; 68:4541-50. [PubMed: 18559498]

8. Peng L, Ran YL, Hu H, et al. Secreted LOXL2 is a novel therapeutic target that promotes gastric cancer metastasis via the Src/FAK pathway. Carcinogenesis. 2009; 30:1660-9. [PubMed: 19625348]

9. Sano M, Aoyagi K, Takahashi H, et al. Forkhead box A1 transcriptional pathway in KRT7expressing esophageal squamous cell carcinomas with extensive lymph node metastasis. Int J Oncol. 2010; 36:321-30. [PubMed: 20043065]

10. Brekhman V, Neufeld G. A novel asymmetric 3D in-vitro assay for the study of tumor cell invasion. BMC Cancer. 2009; 9:415. [PubMed: 19948022]

11. Hollosi P, Yakushiji JK, Fong KS, Csiszar K, Fong SF. Lysyl oxidase-like 2 promotes migration in noninvasive breast cancer cells but not in normal breast epithelial cells. Int J Cancer. 2009; 125:318-27. [PubMed: 19330836] 
12. Kirschmann DA, Seftor EA, Fong SF, et al. A molecular role for lysyl oxidase in breast cancer invasion. Cancer Res. 2002; 62:4478-83. [PubMed: 12154058]

13. Akiri G, Sabo E, Dafni H, et al. Lysyl oxidase-related protein-1 promotes tumor fibrosis and tumor progression in vivo. Cancer Res. 2003; 63:1657-66. [PubMed: 12670920]

14. Sebban S, Davidson B, Reich R. Lysyl oxidase-like 4 is alternatively spliced in an anatomic sitespecific manner in tumors involving the serosal cavities. Virchows Arch. 2009; 454:71-9. [PubMed: 19015874]

15. van de Vijver MJ, He YD, van't Veer LJ, et al. A gene-expression signature as a predictor of survival in breast cancer. N Engl J Med. 2002; 347:1999-2009. [PubMed: 12490681]

16. Nicolau M, Tibshirani R, Borresen-Dale AL, Jeffrey SS. Disease-specific genomic analysis: identifying the signature of pathologic biology. Bioinformatics. 2007; 23:957-65. [PubMed: 17277331]

17. Aslakson CJ, Miller FR. Selective events in the metastatic process defined by analysis of the sequential dissemination of subpopulations of a mouse mammary tumor. Cancer Res. 1992; 52:1399-405. [PubMed: 1540948]

18. Erler JT, Weaver VM. Three-dimensional context regulation of metastasis. Clin Exp Metastasis. 2009; 26:35-49. [PubMed: 18814043]

19. Palamakumbura AH, Trackman PC. A fluorometric assay for detection of lysyl oxidase enzyme activity in biological samples. Anal Biochem. 2002; 300:245-51. [PubMed: 11779117]

20. Guy CT, Cardiff RD, Muller WJ. Induction of mammary tumors by expression of polyomavirus middle T oncogene: a transgenic mouse model for metastatic disease. Mol Cell Biol. 1992; 12:954-61. [PubMed: 1312220]

21. Erler JT, Bennewith KL, Nicolau M, et al. Lysyl oxidase is essential for hypoxia-induced metastasis. Nature. 2006; 440:1222-6. [PubMed: 16642001]

22. Bedogni B, O'Neill MS, Welford SM, et al. Topical treatment with inhibitors of the phosphatidylinositol 3'-kinase/Akt and Raf/mitogen-activated protein kinase kinase/extracellular signal-regulated kinase pathways reduces melanoma development in severe combined immunodeficient mice. Cancer Res. 2004; 64:2552-60. [PubMed: 15059911]

23. Gadea G, Sanz-Moreno V, Self A, Godi A, Marshall CJ. DOCK10-mediated Cdc42 activation is necessary for amoeboid invasion of melanoma cells. Curr Biol. 2008; 18:1456-65. [PubMed: 18835169]

24. Erler JT, Bennewith KL, Cox TR, et al. Hypoxia-induced lysyl oxidase is a critical mediator of bone marrow cell recruitment to form the premetastatic niche. Cancer Cell. 2009; 15:35-44. [PubMed: 19111879]

25. Sleeman KE, Kendrick H, Ashworth A, Isacke CM, Smalley MJ. CD24 staining of mouse mammary gland cells defines luminal epithelial, myoepithelial/basal and non-epithelial cells. Breast Cancer Res. 2006; 8:R7. [PubMed: 16417656]

26. Vadasz Z, Kessler O, Akiri G, et al. Abnormal deposition of collagen around hepatocytes in Wilson's disease is associated with hepatocyte specific expression of lysyl oxidase and lysyl oxidase like protein-2. J Hepatol. 2005; 43:499-507. [PubMed: 16023247]

27. Lin EY, Jones JG, Li P, et al. Progression to malignancy in the polyoma middle T oncoprotein mouse breast cancer model provides a reliable model for human diseases. Am J Pathol. 2003; 163:2113-26. [PubMed: 14578209]

28. Friedl P, Wolf K. Tube travel: the role of proteases in individual and collective cancer cell invasion. Cancer Res. 2008; 68:7247-9. [PubMed: 18794108]

29. Offenberg H, Brunner N, Mansilla F, Torben F Orntoft, Birkenkamp-Demtroder K. TIMP-1 expression in human colorectal cancer is associated with TGF-B1, LOXL2, INHBA1, TNF-AIP6 and TIMP-2 transcript profiles. Mol Oncol. 2008; 2:233-40. [PubMed: 19383344]

30. Pazzaglia L, Ponticelli F, Magagnoli G, et al. Activation of metalloproteinases- 2 and -9 by interleukin-1alpha in S100A4-positive liposarcoma cell line: correlation with cell invasiveness. Anticancer Res. 2004; 24:967-72. [PubMed: 15161051]

31. Pritchard SC, Nicolson MC, Lloret C, et al. Expression of matrix metalloproteinases 1, 2, 9 and their tissue inhibitors in stage II non-small cell lung cancer: implications for MMP inhibition therapy. Oncol Rep. 2001; 8:421-4. [PubMed: 11182067] 
32. Benaud C, Dickson RB, Thompson EW. Roles of the matrix metalloproteinases in mammary gland development and cancer. Breast Cancer Res Treat. 1998; 50:97-116. [PubMed: 9822215]

33. Green KA, Lund LR. ECM degrading proteases and tissue remodelling in the mammary gland. Bioessays. 2005; 27:894-903. [PubMed: 16108064]

34. Ma XJ, Dahiya S, Richardson E, Erlander M, Sgroi DC. Gene expression profiling of the tumor microenvironment during breast cancer progression. Breast Cancer Res. 2009; 11:R7. [PubMed: 19187537]

35. Sleeman KE, Kendrick H, Robertson D, Isacke CM, Ashworth A, Smalley MJ. Dissociation of estrogen receptor expression and in vivo stem cell activity in the mammary gland. J Cell Biol. 2007; 176:19-26. [PubMed: 17190790]

36. Jemal A, Siegel R, Ward E, Hao Y, Xu J, Thun MJ. Cancer statistics, 2009. CA Cancer J Clin. 2009; 59:225-49. [PubMed: 19474385]

37. Hortobagyi GN. Treatment of breast cancer. N Engl J Med. 1998; 339:974-84. [PubMed: 9753714]

38. Levental KR, Yu H, Kass L, et al. Matrix crosslinking forces tumor progression by enhancing integrin signaling. Cell. 2009; 139:891-906. [PubMed: 19931152]

39. Kaplan RN, Riba RD, Zacharoulis S, et al. VEGFR1-positive haematopoietic bone marrow progenitors initiate the pre-metastatic niche. Nature. 2005; 438:820-7. [PubMed: 16341007]

40. Barry-Hamilton V, Spangler R, Marshall D, et al. Allosteric inhibition of lysyl oxidase-like-2 impedes the development of a pathologic microenvironment. Nat Med. 16:1009-17. [PubMed: 20818376]

41. Radisky DC, Hartmann LC. Mammary involution and breast cancer risk: transgenic models and clinical studies. J Mammary Gland Biol Neoplasia. 2009; 14:181-91. [PubMed: 19404726]

42. McDaniel SM, Rumer KK, Biroc SL, et al. Remodeling of the mammary microenvironment after lactation promotes breast tumor cell metastasis. Am J Pathol. 2006; 168:608-20. [PubMed: 16436674]

43. O’Brien J, Lyons T, Monks J, et al. Alternatively activated macrophages and collagen remodeling characterize the postpartum involuting mammary gland across species. Am J Pathol. 2010; 176:1241-55. [PubMed: 20110414]

44. Stein T, Salomonis N, Nuyten DS, van de Vijver MJ, Gusterson BA. A mouse mammary gland involution mRNA signature identifies biological pathways potentially associated with breast cancer metastasis. J Mammary Gland Biol Neoplasia. 2009; 14:99-116. [PubMed: 19408105]

45. Aprelikova O, M Wood, Tackett S, Chandramouli GV, Barrett JC. Role of ETS transcription factors in the hypoxia-inducible factor-2 target gene selection. Cancer Res. 2006; 66:5641-7. [PubMed: 16740701]

46. Saito H, Papaconstantinou J, Sato H, Goldstein S. Regulation of a novel gene encoding a lysyl oxidase-related protein in cellular adhesion and senescence. J Biol Chem. 1997; 272:8157-60. [PubMed: 9079631]

47. Sorlie T, Tibshirani R, Parker J, et al. Repeated observation of breast tumor subtypes in independent gene expression data sets. Proc Natl Acad Sci U S A. 2003; 100:8418-23. [PubMed: 12829800]

48. Weigelt B, Horlings HM, Kreike B, et al. Refinement of breast cancer classification by molecular characterization of histological special types. J Pathol. 2008; 216:141-50. [PubMed: 18720457] 
A

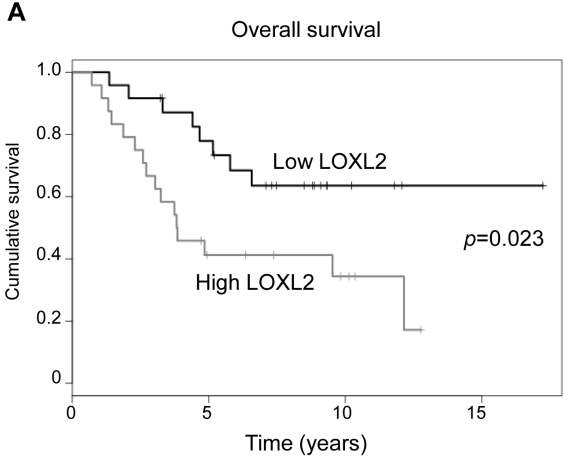

B

Metastasis-free survival

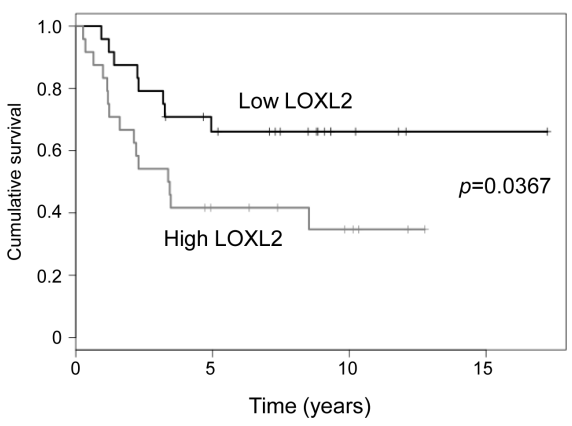

Figure 1. LOXL2 correlates with metastasis and survival

Kaplan-Meier survival curves based on analysis of a previously published microarray dataset from breast cancer patients $(n=295)$ showing $(\mathbf{A})$ overall survival and $(\mathbf{B})$ metastasisfree survival of patients with ER- tumors $(n=72)$ separated into low and high LOXL2 expression. 
A

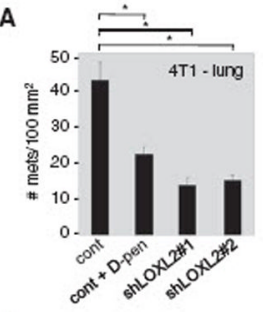

B

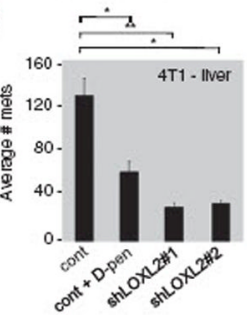

c

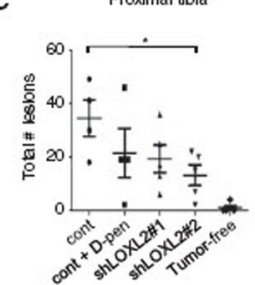

D

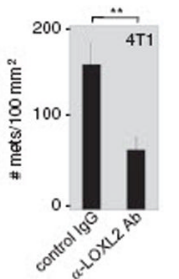

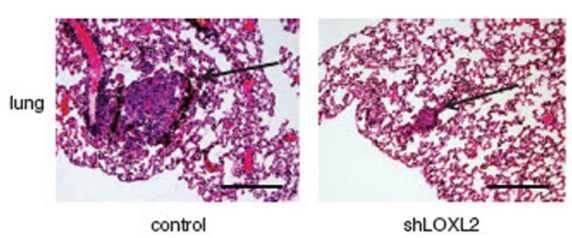

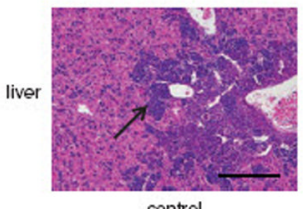

control

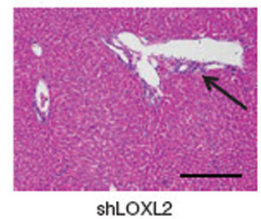

Distal temur
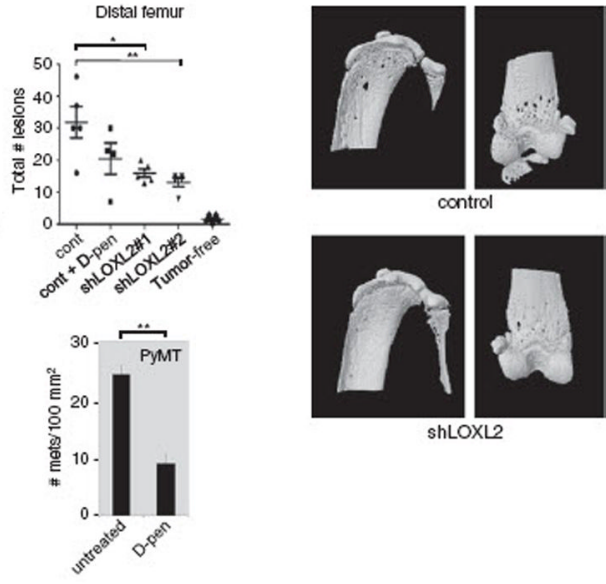

Figure 2. LOXL2 enables metastases in vivo

(A) Quantification of lung metastases formed after orthotopic injection of 4T1control (cont; wild-type or expressing a scrambled control shRNA) and shLOXL2 cells. Mice were either untreated or treated with D-penicillamine(D-pen). At least $n=9$ mice per group.

Representative images are shown in right panel. Scale bar, $200 \mu \mathrm{m}$.

(B) Quantification of liver metastases in mice bearing 4T1control or shLOXL2 orthotopic tumors. Representative images are shown in right panel. Scale bar, $200 \mu \mathrm{m}$.

(C) Quantification of bone metastases in mice bearing 4T1control or shLOXL2 orthotopic tumors. Representative CT images are shown in right panel.

(D) Left panel: Quantification of lung metastases formed after orthotopic injection of 4T1 cells. Mice were treated with IgG or LOXL2-specific antibody. N=5 mice per group. Right panel: Quantification of lung metastases formed in MMTV-PyMT transgenic mice. Mice were either untreated or treated with $\mathrm{D}$-pen. $\mathrm{N}=14$ mice per group. 
A
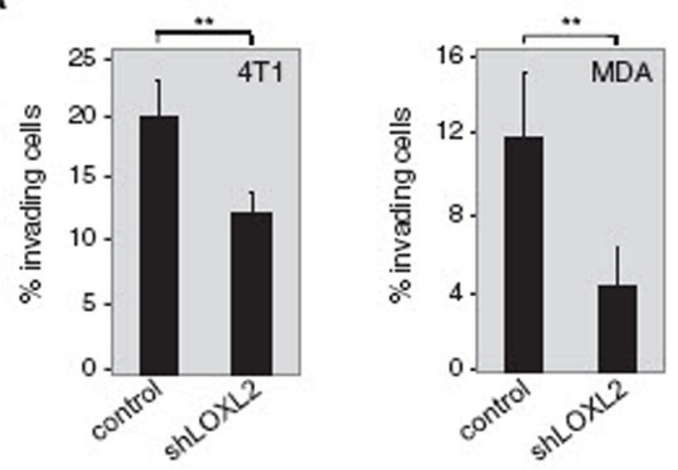

B
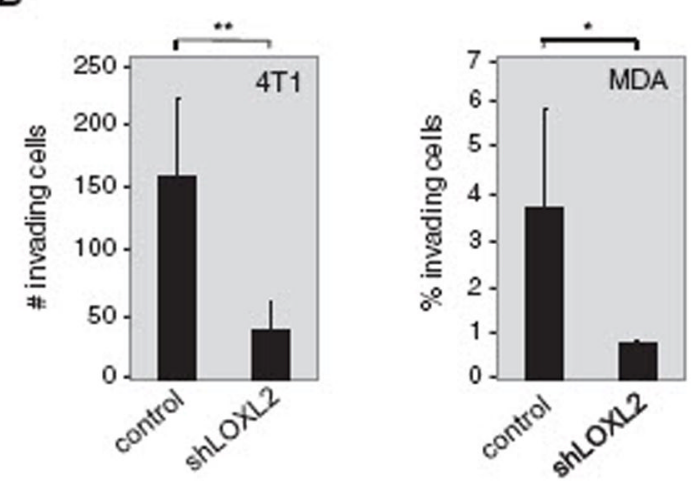

Figure 3. LOXL2 increases in vitro invasion

Quantification of 4T1 or MDA-MB-231control (wild-type or expressing a scrambled control shRNA) and shLOXL2 breast cancer cells invading through (A) collagen or (B) Matrigel towards a chemoattractant. 

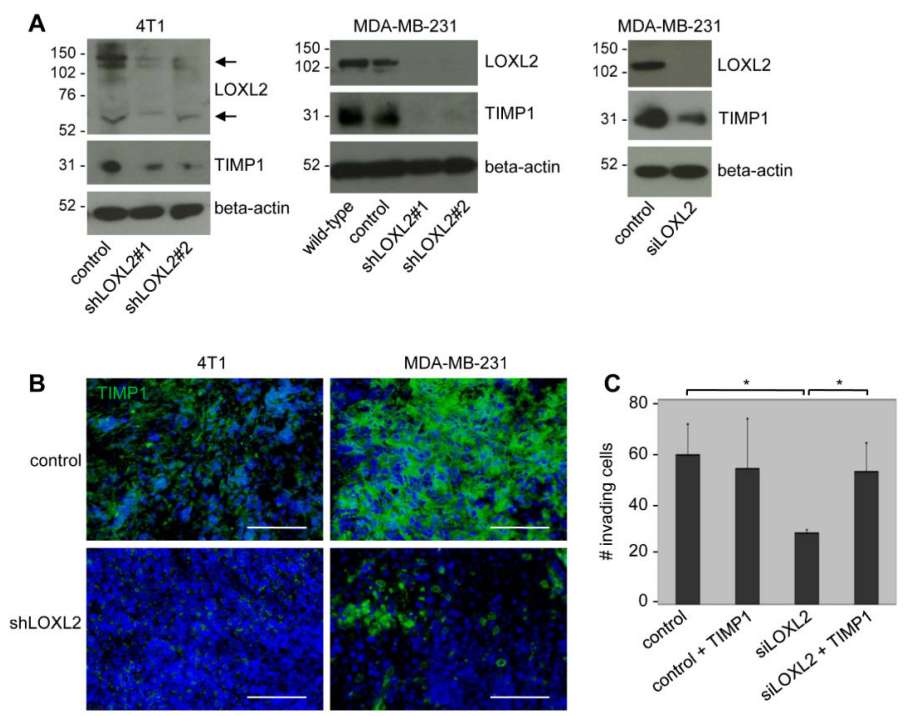

D
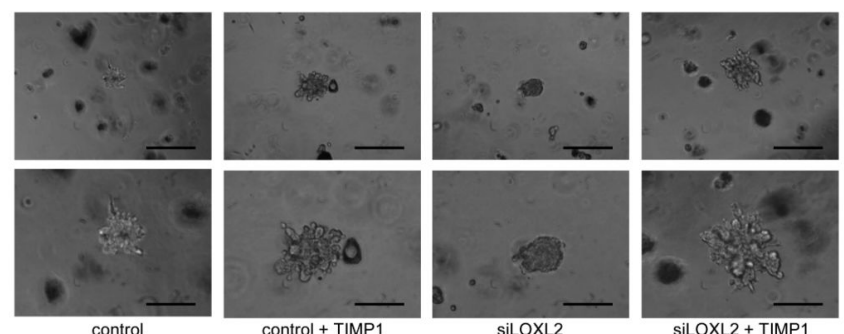

Figure 4. LOXL2 regulates TIMP1

(A) Western blot analysis of LOXL2 protein expression and TIMP1 in concentrated CM from 4T1/MDA-MB-231 cells expressing control or LOXL2 shRNA constructs, or transfected with siLOXL2 (compared with mock-transfected control).

(B) Immunofluorescent images of tumor sections from mice bearing 4T1/MDA-

MB-231control or shLOXL2 tumors stained with TIMP1(green) and DAPI(blue). Scale bar, $100 \mu \mathrm{m}$.

(C) Invasive branching structures formed by MDA-MB-231control or siLOXL2 cells grown in 3D Matrigel, treated \pm recombinant human TIMP1. Scale bar, upper panels; $500 \mu \mathrm{m}$, lower

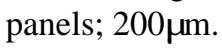


A

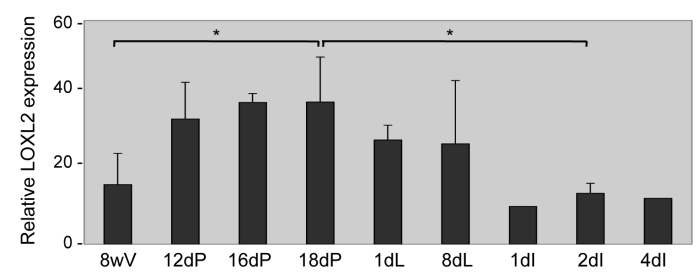

B

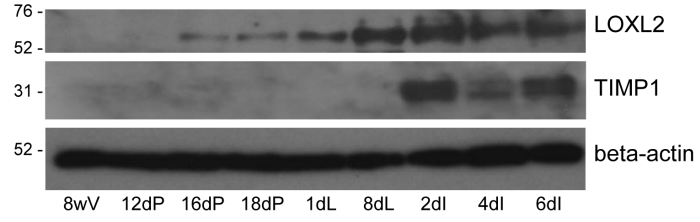

C

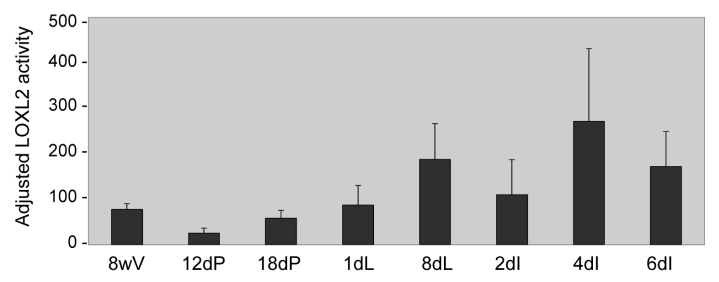

Figure 5. High LOXL2 expression and activity during mammary gland involution

(A) QRT-PCR analysis of LOXL2 mRNA expression levels in FVB mouse mammary gland samples at different stages of development ( $\mathrm{VV}=$ week virgin, $\mathrm{dP}=$ day pregnancy, $\mathrm{dL}=\mathrm{day}$ lactation, $\mathrm{dI}=$ day involution). $\mathrm{N}=3$ mice per time-point.

(B) Western blot analysis of LOXL2 and TIMP1 protein expression in lysates prepared from mice in(A).

(C) LOXL2 enzymatic activity in blood serum from mice in(A). N=3 mice per time-point. 
A
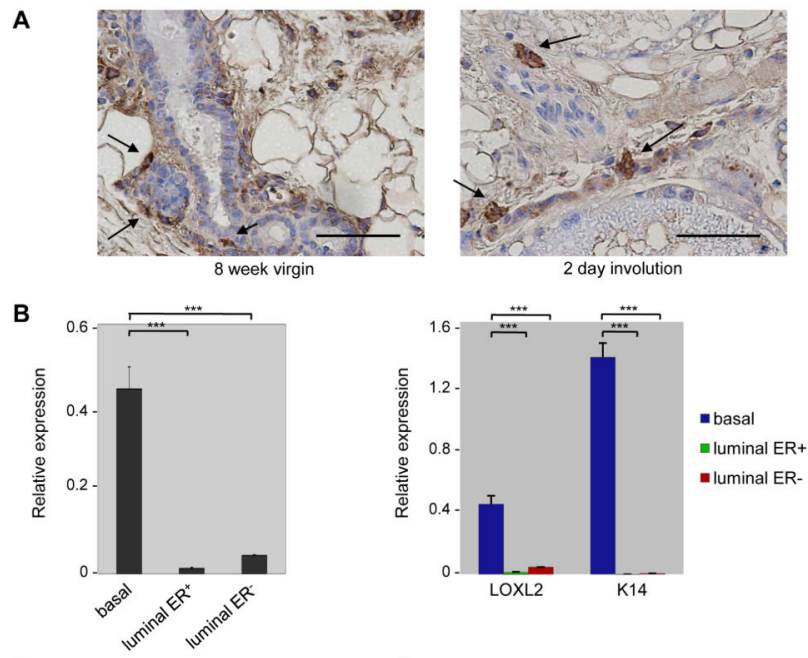

C

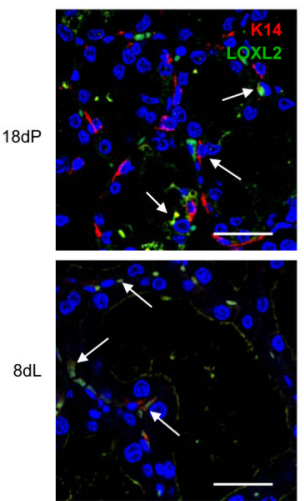

D
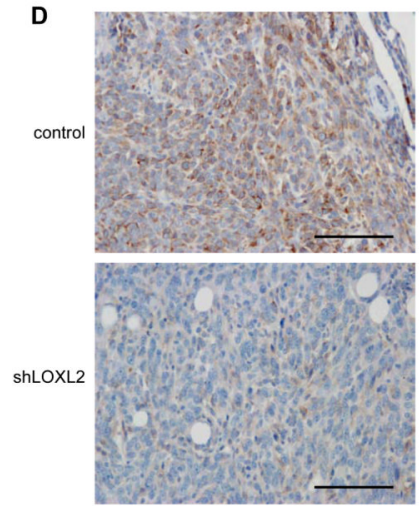

Figure 6. LOXL2 is expressed in stromal and basal epithelial cells

(A) Mammary gland sections from 8 week-old virgin and 2 day involution FVB mice

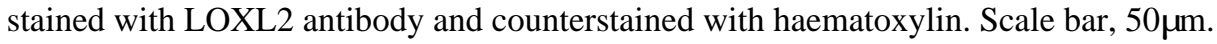

(B) QRT-PCR analysis of LOXL2 and K14 mRNA expression in sorted mammary epithelial cell populations (basal/myoepithelial $=\mathrm{CD} 24^{\text {low }} / \mathrm{Sca}-1^{-}$; luminal ER+=CD24 ${ }^{\text {high }} / \mathrm{Sca}-1^{+}$; luminal ER-=CD24 ${ }^{\mathrm{high}} / \mathrm{Sca}-1^{-}$).

(C) Mammary gland sections from 18 day pregnant(18dP) and 8 day lactating(8dL) FVB mice stained with LOXL2(green) and K14(red) antibodies and counterstained with DAPI(blue). Scale bar, 30 $\mu \mathrm{m}$.

(D) 4T1 tumor sections stained with K14 antibody and counterstained with haematoxylin. Scale bar, $200 \mu \mathrm{m}$. 